\title{
Colored diamonds from Anabar alluvial deposits, Western Yakutia. Classification on the base of spectroscopic features and colorimetrical data
}

\author{
Roman Serov and Yuri Shelementiev \\ Moscow State University Gemological Centre, Moscow, Russia
}

Anabar diamond alluvial deposits in Western Yakutia are exploited by Alrosa for some last years. Diamonds from those deposits have higher percentage of colored crystals. Varieties of main and additional colors of those diamonds don't allow classify it by color in easy and linear way. But a reasonable color classification of mined diamonds is needed for their commercial sorting and evaluation.

We undertake visible spectroscopic research of Anabar colored diamonds from two alluvial localities in order to introduce color grading system based on color origin features and colorimetrical data.

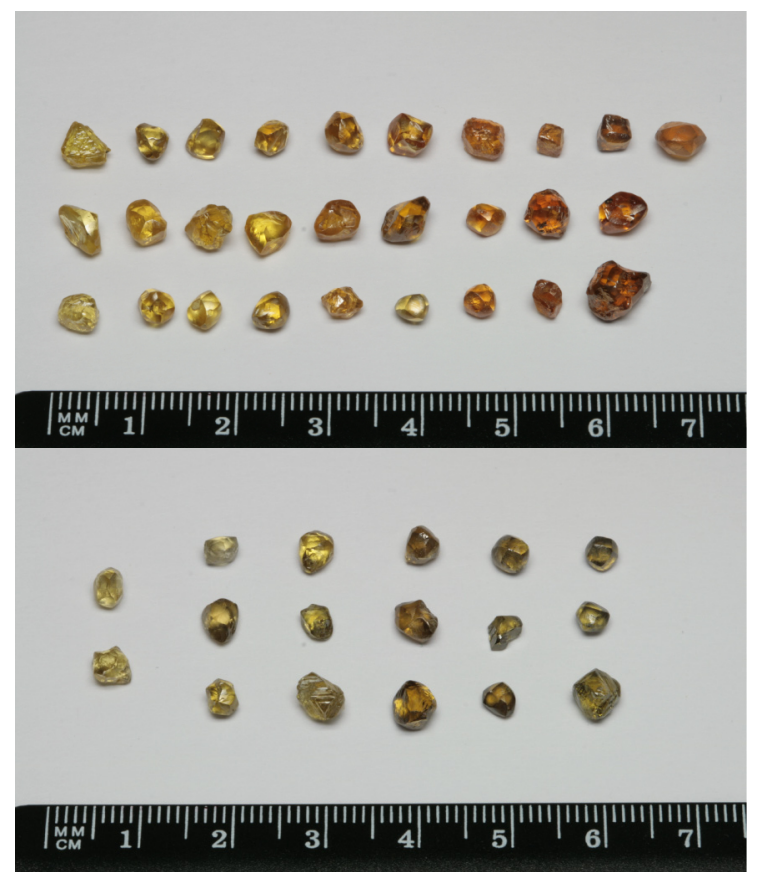

Fig. 1 On the top - yellow to yellow-orange diamonds $\mathrm{Ia}+\mathrm{Ib}$ type (first group). Below - yellow to greenishyellow diamonds Ia+Ib type (second group)

From 247 colored rough diamonds we select 120 samples with shape and surface quality that allow us obtain the reliable VIS and IR spectroscopic data. Absorption spectra in IR region from 400 to $4000 \mathrm{~cm}^{-1}$ were collected. Concentrations of nitrogen-related centers were calculated using IR-data.
According on IR spectroscopy all diamonds can be separated into three types (see fig. 2):

- Ia+Ib. Such diamonds have specific absorption related with $\mathrm{A}$ and $\mathrm{C}$ defects. $76 \%$ of investigated colored diamonds have such type.

- IaA+B. $23 \%$ of investigated diamonds don't have any lines in IR spectra related with $\mathrm{C}$-defects, but have lines related with $\mathrm{A}, \mathrm{B} 1$ and $\mathrm{B} 2$ defects.

- IIa. This diamonds were very rare, only two samples have no nitrogen-related absorption in IR-spectra.
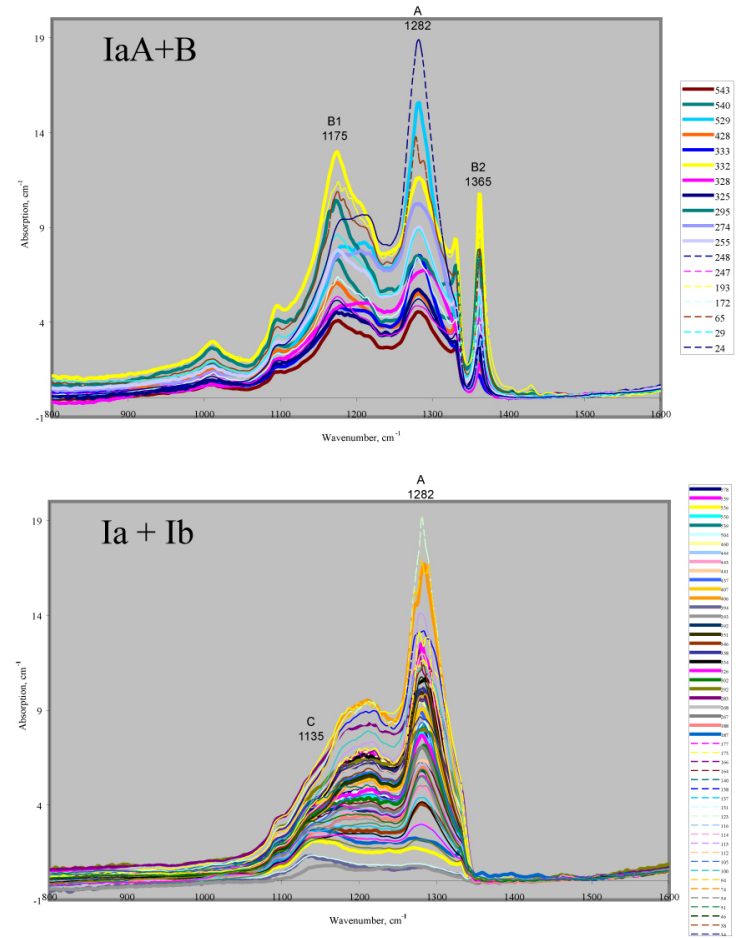

Fig. 2 Some IR spectra of investigated diamonds

Absorption spectra in visible region 370 to $770 \mathrm{~nm}$ were adjusted by considering all possible light losses and corrected for further quantitative studies. After that diamond plates with the same spectra were modeled in DiamCalc software (fig. 3).

Analysis of obtained VIS spectra allows us separate all diamonds into 6 different color types based on presence various color origin features (fig. 5). 


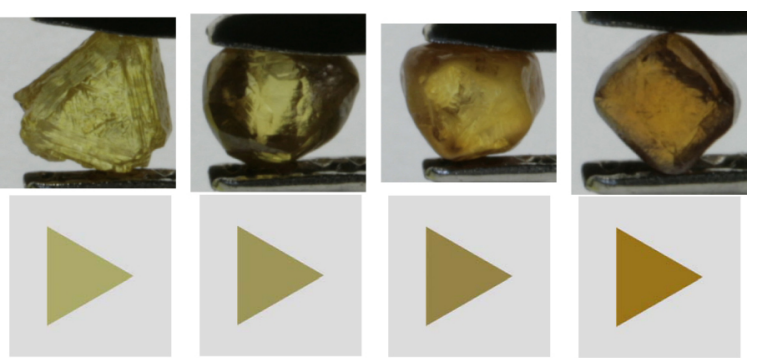

Fig. 3 Comparison with diamond photos and modeled by absorption spectra plate with the same thickness

First two color groups correspond to $\mathrm{Ia}+\mathrm{Ib}$ type by IR spectroscopy. Third, forth and fifth groups to IaA+B type. All pink diamonds from sixth group belong to IIa type.

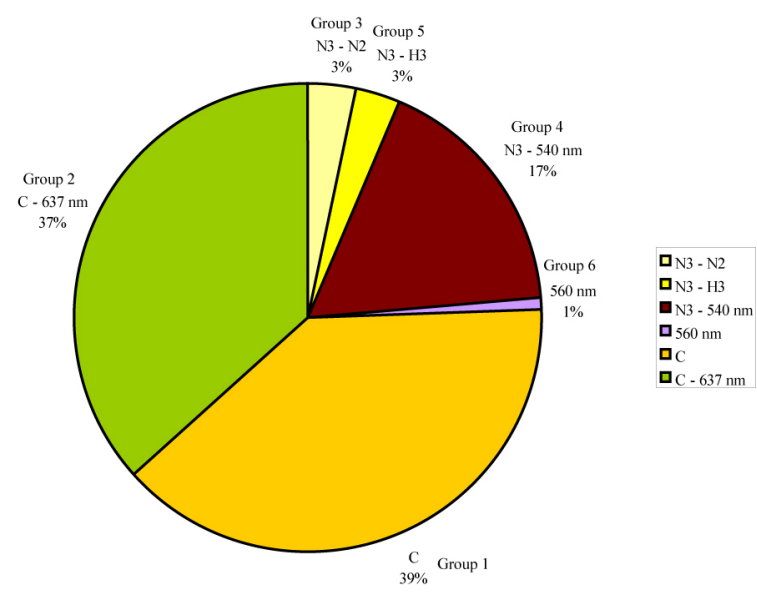

Fig. 4 Percentage of different color groups

- First group contains yellow to yellow-orange diamonds that are characterized by $\mathrm{C}$-centers related absorption only. No N3 or H3 defects lines appeared on these group diamonds spectra.

- Second group has $(\mathrm{N}-\mathrm{V})^{+}$centers absorption on 637 $\mathrm{nm}$ (Zaitsev, 2001) in addition to C-centers that caused greenish-yellow color for diamonds. Formation of N-V centers is related with C-centers concentration. So only in saturated diamonds of that group $(\mathrm{N}-\mathrm{V})^{+}$centers were clearly visible.

- Nitrogen contained as N3 (415 nm) and N2 (476 $\mathrm{nm})$ centers gives light yellow to yellow color for diamonds from the third group. This diamonds also shown blue LW UV luminescence.

- Forth group have light brown to deep brown and reddish-brown color caused by deformation-related absorption (broad bands on 470 and $540 \mathrm{~nm}$ ).

- Fifth group diamonds have N3 and H3 (503 nm) H4 (496 nm) centers in VIS spectra. These diamonds have yellow color with green hue. Diamonds from this group show greenish-yellow LW and SW UV luminescence.

- Diamonds from sixth group are very rare and have pink color concerned with $560 \mathrm{~nm}$ broad band absorption.

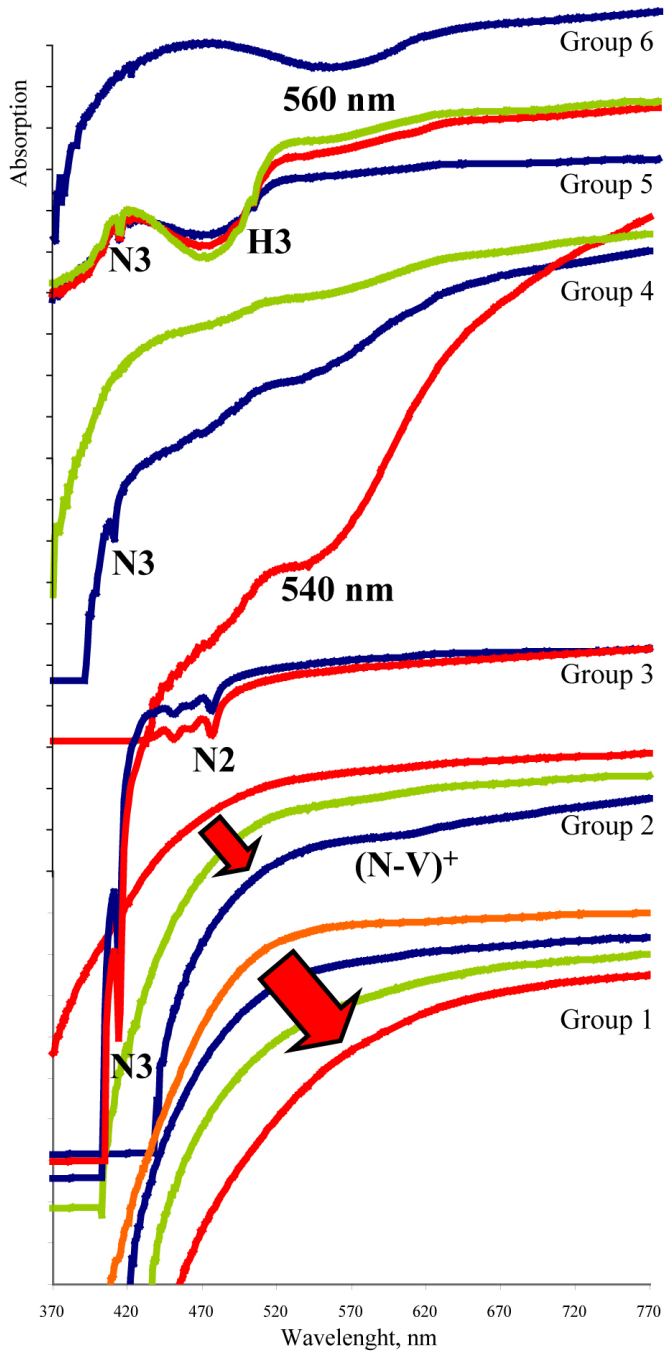

Fig. 5 Characteristic VIS spectra for diamond coloration types

Color coordinates xy for all stones were located on locus diagram. This permits us to show gradual color change because of concentration of color centers and, as a result, gradual modification of stone's hue and other color components (fig. 6).

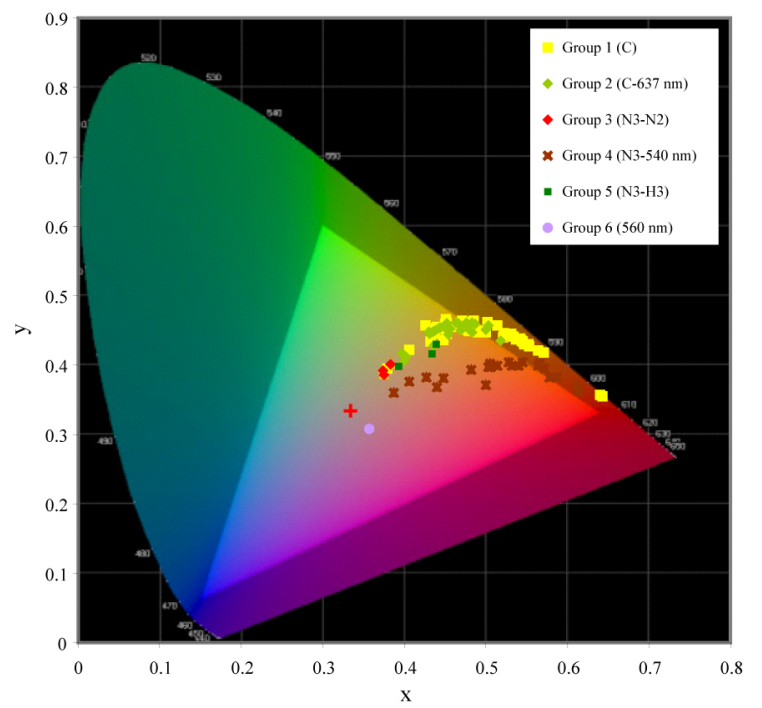

Fig. 6 Places of investigated diamonds color in $x y$ color space 
Even better separation of diamonds onto 6 color groups is possible in three-dimensional representation with introduction of the third color coordinate Z (fig. 7).

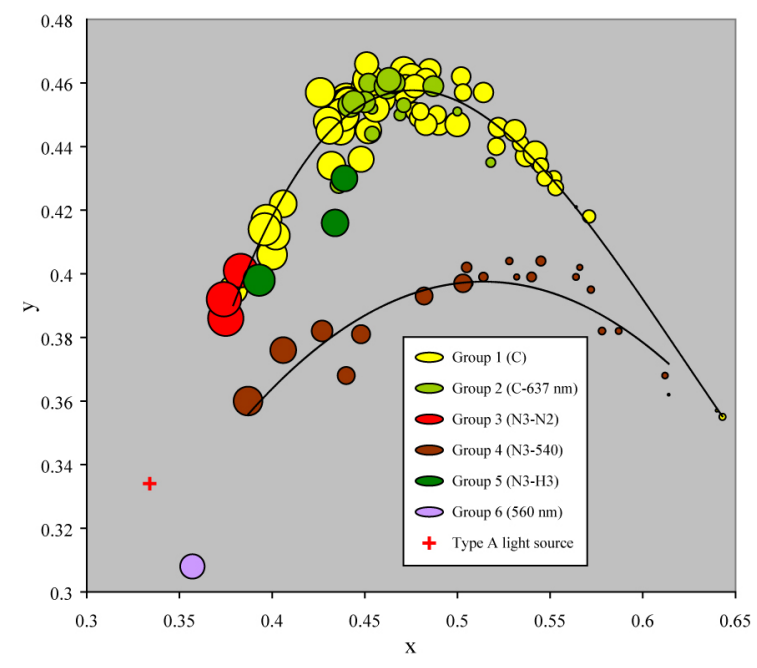

Fig. 7 Places of investigated diamonds color in $x y Z$ color space. $\mathrm{Z}$ coordinate value is related with circle size

On that diagram two trends are clearly visible. Trend for yellow circles correspond to color change of group 1 diamonds from yellow to orange-yellow. We compared color saturation of that stones calculated on the base of VIS spectra in DiamCalc and concentration of C-defect calculated by IR spectra. And find good linear correlation between those values. So it can be affirmed that in natural colored diamonds Ia+Ib type yellow to yellow-orange color saturation is linearly depends on C-defects concentration calculated by IRspectra. So the trend for that diamonds group in color space can be normalized by $\mathrm{C}$-centre concentration (fig. 8).

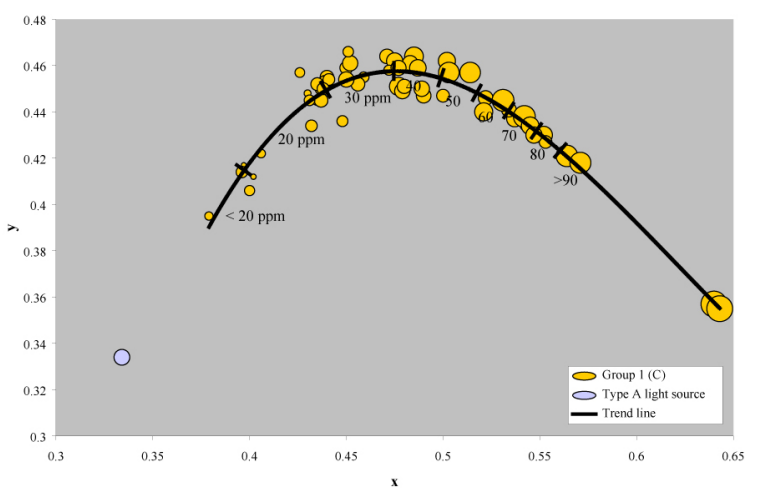

Fig. 8 Correlation between color and C-defect concentration for type $\mathrm{Ia}+\mathrm{Ib}$ diamonds. Circle size corresponds to $\mathrm{C}$-centre concentration

Also on fig. 7 trend for diamonds of group 4 (brown circles) is conspicuous. This trend shows color change from light brown to deep brown and reddish-brown for that diamonds. No correlation between color saturation of that stones and nitrogen-related defects has been found. It was believed that $540 \mathrm{~nm}$ absorption bands related with plastic deformation of diamond and higher level of deformation can cause higher absorption (Bokii et al, 1986).

On fig. 9 Taylor-Milledge diagram for diamond crystals from groups 4 and 5 is shown. Brown diamonds from group 4 occupy a large area, so it can be supposed that such diamonds come to the alluvial deposit from multiple sources. Otherwise greenishyellow diamonds from group 5 are located in fixed area. So we can suppose one initial source for that diamonds.

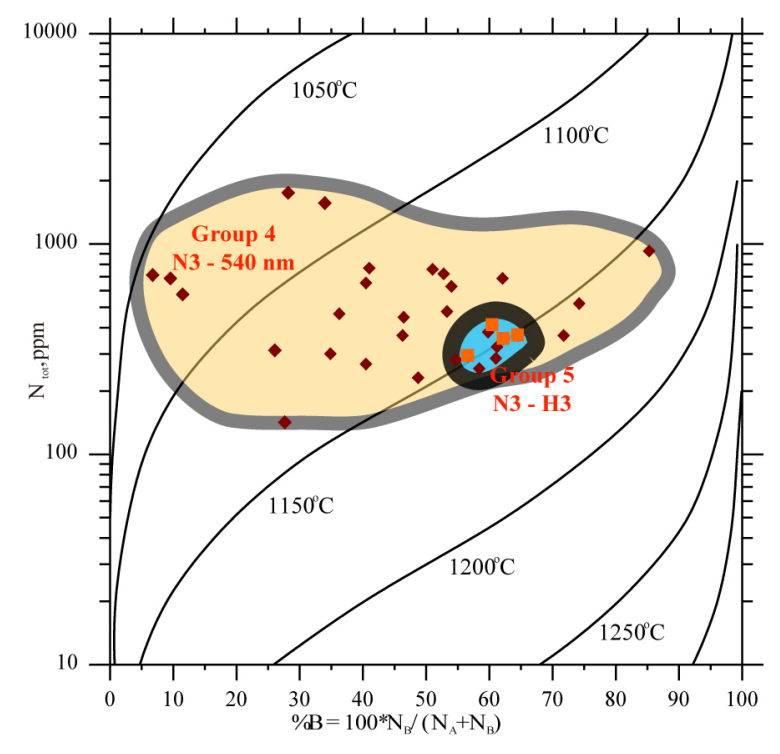

Fig. 9 Taylor-Milledge diagram (Taylor, Milledge 1995) for diamond crystals from groups 4 and 5. Isotherm curves are for $3 \mathrm{Ga}$

Combination of color computer modeling based on optical spectroscopy and computer diamond planning technology allows predicting color appearance of future polished diamonds and certain fancy color grade.

Selected diamonds with known spectroscopic and colorimetric data can be used as master stones for further color grading of colored diamonds.

This research project was done in cooperation with Alrosa company.

\section{References}

Bokii, G.B., Bezrukov, G. N., Klyuev, Yu. A., Naletov, A. M., Nepsha, V. I., 1986. Natural and Synthetic Diamonds. Moscow: Nauka, (In Russian).

Taylor, W.R., Milledge H.J, 1995. Nitrogen aggregation character, thermal history and stable isotope composition of some xenolith-derived diamonds from Roberts Victor and Finch. In: Sixth International Kimberlite Conference Extended Abstract, Novosibirsk, August, 620-622.

Zaitsev, A.M, 2001. Optical Properties of Diamond: A Data Handbook. Berlin Springer Verlag, 502. 